\title{
Rainbow universe
}

\author{
Yi Ling ${ }^{1,2 *}$ \\ 1 Center for Gravity and Relativistic Astrophysics, Nanchang University, 330047, China and \\ 2 CCAST (World Laboratory), P.O. Box 8730, Beijing 100080, China
}

\begin{abstract}
The formalism of rainbow gravity is studied in a cosmological setting. We consider the very early universe which is radiation dominated. A novel treatment in our paper is to look for an "averaged" cosmological metric probed by radiation particles themselves. Taking their cosmological evolution into account, we derive the modified Friedmann-Robertson-Walker(FRW) equations which is a generalization of the solution presented by Magueijo and Smolin. Based on this phenomenological cosmological model we argue that the spacetime curvature has an upper bound such that the cosmological singularity is absent. These modified $F R W$ equations can be treated as effective equations in the semi-classical framework of quantum gravity and its analogy with the one recently proposed in loop quantum cosmology is also discussed.
\end{abstract}

\section{INTRODUCTION}

Any theory aiming to reconcile general relativity and quantum mechanics is characterized by the presence of the Planck length $\left(l_{p}=\sqrt{\hbar G / c^{3}}\right)$, which is the combination of the gravitational constant, the Planck constant and the speed of light. It has been long believed that this should be the minimum length scale that one can observe or take measurement in laboratory. In a cosmological setting it is also conjectured that the Planck length would provide a bound to the curvature of spacetime such that the big-bang singularity could be avoided in the framework of quantum gravity.

In recent years the semi-classical or phenomenological effect of quantum gravity has also been greatly investigated 1, 2 , 3, 4, 5, 6, 7, 8]. This is an essential step to link the fundamental quantum theory of gravity which is under exploration and the classical general relativity which however has proved to describe our low energy world quite well. Particularly it is proposed that it may be responsible for some puzzles occurring in recent astronomical and cosmological observations, such as the threshold anomalies of ultra high energy cosmic rays and Tev photons [9, 10, 11, 12, 13, 14, 15]. Then in this direction a key issue arises, namely how to construct a semiclassical or effective theory of quantum gravity where the Planck length may play a fundamental role but without violating the principle of relativity. Among several candidates, doubly special relativity is supposed to be such a framework to implement this scheme. It is a deformed version of conventional special relativity with two universal constants which are the same for all inertial observers, one being the speed of light while the other the Planck length or Planck energy. This can be accomplished by a non-linear Lorentz transformation in momentum space, which leads to a deformed Lorentz symmetry such that the usual energy-momentum relations or dispersion relations in special relativity may be modified with correc-

*Electronic address: yling@ncu.edu.cn tions in the order of Planck length. Nevertheless, in the framework of doubly special relativity the definition of the dual position space is not trivial due to the nonlinearity of the Lorentz transformation. To overcome this difficulty, Magueijo and Smolin proposed in [16] that maybe there is no single spacetime background when the effect of moving probes on geometry is taken into account. Instead, it is replaced by a one parameter family of metrics which depends on the energy of these test particles, forming a "rainbow" metric. More explicitly, corresponding to each modified dispersion relation, a dual rainbow metric can be constructed. Consequently when incorporating curved spacetime the connection and curvature are energy dependent such that the usual Einstein's equations is modified as a one parameter family of equations. This proposal has received a lot of attention recently and other stimulated work on this formalism can be found in [17, 18, 19, 20, 21, 22, 23].

As specific examples the modified version of $F R W$ solution and Schwarzschild solution to these equations have been presented in [16]. These solutions are derived in a general sense that the probe can be any test particle moving in the spacetime. Thus during the process of solving deformed Einstein's equations, those correction terms in a rainbow metric, though dependent on the energy of probes $\epsilon$, can be treated as a constant independent of spacetime coordinates. In this paper we intend to take the formalism of rainbow gravity as a semi-classical framework of quantum gravity, and study its impact on the evolution of the early universe. Rather than taking any specific measurement into account, we intend to consider the semi-classical effect of radiation particles on the spacetime metric during a longtime process. Then the probe energy appearing in the correction term of the rainbow metric is identified with the one of photons or other sorts of massless particles like gravitons which dominate the very early universe. In this setting the energy of probes obviously varies with the cosmological time. In this paper we take this effect into account and derive a generalized formalism of the modified $F R W$ equations previously presented in [16]. Based on this, we further propose a strategy to define an intrinsic metric for the universe by considering the "average" effect of all radi- 
ation particles. This phenomenological model provides the spacetime curvature an upper bound such that the cosmological singularity is absent in this semi-classical framework. We will end this paper by pointing out the analogy of the modified $F R W$ equations presented here with the one recently proposed in loop quantum cosmology.

\section{REVIEW ON RAINBOW GRAVITY}

We start this section with a quick review on rainbow gravity formalism proposed by Magueijo and Smolin [16]. In deformed or doubly special relativity, the invariant of energy and momentum in general may be realized by a non-linear Lorentz transformation in momentum space, leading to a modified energy-momentum relation or dispersion relations as

$$
\epsilon^{2} f_{1}^{2}(\epsilon, \eta)-p^{2} f_{2}^{2}(\epsilon, \eta)=m_{0}^{2},
$$

where $f_{1}$ and $f_{2}$ are two functions of energy depending on the specific formulation of boost generator and $\eta$ is a dimensionless parameter. Now to incorporate the effect of gravity, they proposed a deformed equivalence principle of general relativity, stating that the free falling observers who make measurements with energy $\epsilon$ will observe the same laws of physics as in modified special relativity. Furthermore, the correspondence principle requires that $f_{1}$ and $f_{2}$ approach to unit as $\epsilon / E_{p} \ll 1$. On the other hand, the position space is defined by requiring that the contraction between momenta and infinitesimal displacement be a linear invariant.

$$
d x^{\mu} p_{\mu}=d t \epsilon+d x^{i} p_{i}
$$

As a result, the dual space is endowed with an energy dependent invariant

$$
d s^{2}=-\frac{1}{f_{1}^{2}(\epsilon, \eta)} d t^{2}+\frac{1}{f_{2}^{2}(\epsilon, \eta)} d x^{2} .
$$

Keep going on and taking the effect of gravity into account, a one parameter family of connections as well as curvature tensors can be constructed from the rainbow metrics, leading to a modified Einstein's field equations

$$
G_{\mu \nu}(\epsilon)=8 \pi G(\epsilon) T_{\mu \nu}(\epsilon)+g_{\mu \nu}(\epsilon) \Lambda(\epsilon),
$$

where Newton's constant as well as the cosmological constant is conjectured to be energy dependent in general as one expects from the viewpoint of renormalization group theory, for instance $G(\epsilon)=g^{2}(\epsilon) G$ and $\Lambda(\epsilon)=h^{2}(\epsilon) \Lambda$.

\section{MODIFIED FRW UNIVERSE}

In this section we derive cosmological solutions to modified Einstein equations (4). The modified FRW metric for a homogeneous and isotropic universe may be expressed in terms of energy independent coordinates as

$$
d s^{2}=-\frac{1}{f_{1}^{2}(\epsilon, \eta)} d t^{2}+\frac{1}{f_{2}^{2}(\epsilon, \eta)} a(t) \gamma_{i j} d x^{i} d x^{j}
$$

where $\gamma_{i j}$ is the spatial metric. For simplicity we consider the spatially flat universe with

$$
K=0 \quad \Lambda=0 \quad \gamma_{i j}=\delta_{i j}
$$

but it is expected to be straightforward extending our analysis to the other cases.

Usually one is free to pick up arbitrary particle as a probe and for any specific operation of measurement its energy $\epsilon$ can be treated as a constant which is independent of spacetime coordinates. However, in early universe of course no such real measurement is taken. Here we intend to apply this mechanism to study the semiclassical effect of radiation particles on the background metric during its evolution which is a longtime process. Then it is natural to take one of radiation particles as the probe. Moreover, in this circumstance the evolution of energy $\epsilon$ with the cosmological time need to be considered, maybe denoted as $\epsilon(t)$. Now taking this into account we derive the modified $F R W$ equations following the standard process in general relativity. For convenience we further take the following ansatz

$$
\eta=1, \quad f_{2}(\epsilon, \eta)=g^{2}(\epsilon)=1
$$

such that the non-vanishing components of the associated connection read as

$$
\Gamma_{00}^{0}=-\frac{\dot{f}}{f}, \quad \Gamma_{i j}^{0}=f^{2} \dot{a} a \delta_{i j}, \quad \Gamma_{0 j}^{i}=\delta_{j}^{i} \frac{\dot{a}}{a} .
$$

Going ahead we obtain the non-trivial components of Riemann tensor as

$$
\begin{aligned}
R_{00 j}^{i} & =\frac{\ddot{a}}{a} \delta_{j}^{i}+\frac{\dot{a} \dot{f}}{a f} \delta_{j}^{i} \\
R_{i 0 j}^{0} & =f^{2} a \ddot{a} \delta_{i j}+f \dot{f} a \dot{a} \delta_{i j} \\
R_{j k m}^{i} & =f^{2} \dot{a}^{2}\left(\delta_{k}^{i} \delta_{j m}-\delta_{m}^{i} \delta_{j k}\right) .
\end{aligned}
$$

Thus the Ricci tensor components are

$$
\begin{aligned}
R_{00} & =3 \frac{\ddot{a}}{a}+3 \frac{\dot{a} \dot{f}}{a f} \\
R_{i j} & =\left[f^{2}\left(a \ddot{a}+2 \dot{a}^{2}\right)+f \dot{f} a \dot{a}\right] \delta_{i j} \\
R & =-6 f^{2}\left(\frac{\ddot{a}}{a}+\frac{\dot{a} \dot{f}}{a f}+\frac{\dot{a}^{2}}{a^{2}}\right)
\end{aligned}
$$

Next we consider the fluid with the energy-momentum tensor

$$
T_{\mu \nu}=\rho u_{\mu} u_{\nu}+P\left(g_{\mu \nu}+u_{\mu} u_{\nu}\right)
$$


where $\rho$ denotes the energy density while $P$ the pressure, and $u_{\mu}$ is defined as

$$
u_{\mu}=\left(f^{-1}, 0,0,0\right)
$$

such that it is a unit vector, namely

$$
u_{\mu} u_{\nu} g^{\mu \nu}=-1 .
$$

Applying this setting to the conservation equation of energy-momentum tensor

$$
\nabla^{\mu} T_{\mu \nu}=0,
$$

we find that

$$
\dot{\rho}=-3 H(\rho+P),
$$

which is the same as the usual one. It is also interesting to notice that this form is universal for all comoving observers, while the connection or the covariant derivative $\nabla(\epsilon)^{\mu}$ is energy dependent and thus the symbols in (8) have been employed when deriving the equation (15).

Now we turn to the modified Einstein equations. Substituting all the terms obtained above into (4) gives rise to the modified FRW equations as

$$
\begin{aligned}
H^{2} & =\frac{8 \pi G}{3} \frac{\rho}{f^{2}} \\
\dot{H} & =-4 \pi G \frac{(\rho+P)}{f^{2}}-H \frac{\dot{f}}{f},
\end{aligned}
$$

where we have introduced the Hubble factor $H=\dot{a} / a$. As in standard cosmology, from above equations we may derive the conservation equation (15), which means only two of these three equations are independent.

At the end of this section we point out that it is straightforward to derive a general version of modified $F R W$ equations when both $f_{1}$ and $f_{2}$ are general functions varying with the cosmological time.

\section{IMPLICATIONS TO THE COSMOLOGICAL SINGULARITY}

In this section we consider the evolution of modified FRW universe. For explicitness we take the function $f$ as

$$
f^{2}=1-l_{p}^{2} \epsilon^{2},
$$

which has also been considered in the context of black hole physics in 21, 24]. One extraordinary property of this function is giving rise to a relation

$$
\epsilon^{2}=\frac{1}{2 l_{p}^{2}}\left(1-\sqrt{1-4 l_{p}^{2} p^{2}}\right),
$$

such that both the momentum and energy are bounded as $p \leq \frac{1}{2 l_{p}}, \epsilon \leq \frac{1}{\sqrt{2} l_{p}}$. As we stated above, rather than picking up any specific particle from the radiation at random, we more intend to take the radiation as an ensemble and consider the "average" effect of radiation particles which dominate the very early universe. Such a scheme has been applied to investigate the thermodynamics of modified black holes as well. For details we refer to [21, 22] and the previous relevant work in 25]. Then the energy appearing in rainbow metric is identified with the statistical mean value of all massless radiation particles. As a result, the modified dispersion relation varies with the cosmological time

$$
f^{2}=1-l_{p}^{2} \bar{\epsilon}^{2}(t)
$$

Then the conservation equation (15) and the $F R W$ equation (16) read as

$$
\begin{gathered}
\frac{\dot{\rho}}{\rho}=-3(1+\omega) \frac{\dot{a}}{a}, \\
\frac{\dot{a}^{2}}{a^{2}}=\frac{8 \pi G}{3} \frac{\rho}{1-l_{p}^{2} \bar{\epsilon}^{2}} .
\end{gathered}
$$

Before explicitly describing the evolution of the universe subject to equations (20), we still need to know the equations of state of the radiation. As pointed out in references [16, 26], the usual relation of $\rho$ and $P$ would be modified due to the modified dispersion relations, namely $\omega \neq 1 / 3$ exactly. However, as we show in the appendix, when the temperature is below the Planck scale this modification will only provide correction terms in higher orders of the Planck length, which will not change the main picture we illustrate below. Furthermore, from the dynamical point of view we find in this semi-classical framework, the temperature of the universe will also be bounded in the very early epoch, which is about the Planck scale such that the case of $T \gg 1 / l_{p}$, though discussed in our appendix, will not occur in the evolution history of the universe. In hence for our purpose, it is still plausible to identify the energy of radiation particle with the density through the conventional relations

$$
\bar{\epsilon}^{2} \sim \sqrt{\rho} \quad \frac{\dot{\rho}}{\rho} \sim-4 \frac{\dot{a}}{a} .
$$

Thus we have an equation

$$
\frac{l_{p}^{2} \sqrt{1-x}}{x^{2}} \dot{x}=C
$$

where $x=l_{p}^{2} \sqrt{\rho}$ and $C$ is a constant . The solution is

$$
t=\frac{l_{p}^{2}}{C}\left[\frac{\sqrt{1-x}}{x}+\ln \frac{(1-\sqrt{1-x})}{\sqrt{x}}\right]
$$

At the classical limit, namely $l_{p} \rightarrow 0$, it is easy to find that the expression goes back to the usual one $\rho \sim \frac{1}{t^{2}}$, but in early time of the universe $t \rightarrow 0$, we find the density will go to a finite number rather than a divergent disaster

$$
\rho \sim\left(\frac{l_{p}}{l_{p}^{2}+C t}\right)^{4} \sim \frac{1}{l_{p}^{4}}
$$


Thus in this effective theory the cosmological singularity is absent. We stress that the avoidance of singularity in this context is not apparent. Time reparametrization will not change this conclusion and this can be seen from the equation (24).

Of course above consideration based on the identification in (22) is the zeroth approximation and quite sketchy. More quantitative illustration of the evolution may be obtained through numerical simulation based on equations (36) - (39) in the appendix.

\section{DISCUSSIONS AND CONCLUSIONS}

It must be pointed out that the complete understanding on the fate the cosmological singularity calls for a complete quantum theory of gravity. Our analysis presented here, though in a heuristic manner, has shed light on this issue and reflected the important role played by non-perturbative gravity around the Planck scale. One of our motivations discussing the fate of the cosmological singularity in semi-classical regime also comes from recent progress made in loop quantum cosmology, where the exact solutions to quantum equations are remarkably well approximated by a naive extrapolation of some semiclassical effective equations. It is interesting to compare the effective $F W R$ equations from rainbow gravity with those from loop quantum cosmology, where the effective equations have the form [27, 28, 29]

$$
\begin{gathered}
H^{2}=\frac{8 \pi G}{3} \rho\left(1-\frac{\rho}{\rho_{c}}\right) \\
\dot{H}=-4 \pi G(\rho+P)\left(1-2 \frac{\rho}{\rho_{c}}\right)
\end{gathered}
$$

and $\rho_{c} \sim l_{p}^{-4}$. They may be obtained from the eqs. (16) and (16) if we naively require that

$$
f^{2}=\left(1-l_{p}^{4} \epsilon^{4}\right)^{-1}
$$

Therefore, our scheme presented here indicates a potential relation between the rainbow gravity formalism and the effective theory of loop quantum gravity at the semiclassical level 30].

In summary, we have studied the semi-classical effect of radiation particles on the background metric in the framework of rainbow gravity, and obtained the modified $F R W$ equations where the energy of particles varies with the cosmological time. When taking some specific modified dispersion relation into account, we find the evolution picture of the universe in the early era dramatically changes and the cosmological singularity can be avoided. The scheme presented here may also be extended to more general cases, for instance the spatial metric is non-flat and the cosmological constant can also be involved. In particular it is supposed that the scalar field should play an important role in very early universe, its effective theory in the formalism of rainbow gravity as well as the effect of the energy dependence of the Newton's constant is under investigation.

\section{Acknowledgement}

I am grateful to Song He, Bo Hu, Xiang Li, Xiao-qing Wen and Hongbao Zhang for a lot of discussions and helpful suggestions. This work is partly supported by NSFC (No.10405027) and SRF for ROCS, SEM.

\section{APPENDIX: STATISTICS FOR RADIATION PARTICLES WITH MODIFIED DISPERSION RELATIONS}

The thermodynamical feature of radiation particles with modified dispersion relations has been considered previously in the context of non-commutative geometry 26]. Here we start with the general form of modified dispersion relations as considered above

$$
f^{2} \epsilon^{2}-p^{2} c^{2}=0
$$

Thus we have

$$
d p=\frac{f}{c}\left(1+\epsilon \frac{f^{\prime}}{f}\right) d \epsilon .
$$

Then the usual density of states is modified as

$$
\begin{aligned}
g(\epsilon) d \epsilon & =2 \frac{V}{h^{3}} 4 \pi p^{2} d p \\
& =\frac{8 \pi V}{h^{3} c^{3}} f^{3}\left(1+\epsilon \frac{f^{\prime}}{f}\right) \epsilon^{2} d \epsilon .
\end{aligned}
$$

Consequently the following statistical quantities are modified as

$$
\begin{gathered}
\bar{N}=\int \frac{g(\epsilon)}{e^{\epsilon / k T}-1} d \epsilon . \\
\rho=\frac{\bar{U}}{V}=\int \frac{\epsilon}{V} \frac{g(\epsilon)}{e^{\epsilon / k T}-1} d \epsilon . \\
\frac{P V}{k T}=\int-\ln \left(1-e^{-\epsilon / k T}\right) g(\epsilon) d \epsilon .
\end{gathered}
$$

Plugging the modified density of states into the equation (32) we obtain

$$
\begin{aligned}
\bar{N} & =\int \frac{8 \pi V}{h^{3} c^{3}} f^{3}\left(1+\epsilon \frac{f^{\prime}}{f}\right) \frac{\epsilon^{2}}{e^{\epsilon / k T}-1} d \epsilon \\
& =\frac{8 \pi V}{h^{3} c^{3}} \int_{0}^{\frac{1}{\sqrt{2} l_{p}}} \frac{\epsilon^{2}\left(1-l_{p}^{2} \epsilon^{2}\right)^{\frac{1}{2}}\left(1-2 l_{p}^{2} \epsilon^{2}\right)}{e^{\epsilon / k T}-1} d \epsilon .
\end{aligned}
$$


Now we consider above integrations with the specific function given by (17). For low temperature, we may redefine $x \equiv \frac{\epsilon}{k T}$ and $\lambda \equiv l_{p} k T \ll 1$, such that the integral may be approximately evaluated as

$$
\bar{N} \propto V T^{3}\left(1-\alpha l_{p}^{2} T^{2}+\ldots\right) .
$$

Similarly we can get the results for the density and the pressure

$$
\begin{aligned}
& \rho \propto \sigma T^{4}\left(1-\beta l_{p}^{2} T^{2}+\ldots\right), \\
& P \propto \frac{1}{3} \rho\left(1-\gamma l_{p}^{2} T^{2}+\ldots\right),
\end{aligned}
$$

providing modifications to both Stefan-Boltzmann law and the equation of state, where the parameters may approximately evaluated as $\alpha=25.88, \beta=46.95$ and $\gamma=28.17$. As a result, we notice that

$$
\bar{\epsilon}=\frac{\rho V}{\bar{N}} \propto k T\left(1+\delta l_{p}^{2} T^{2}+\ldots\right) .
$$

For high temperature $\lambda \gg 1$, however, these relations will change greatly. It can be found that

$$
\bar{N} \propto \frac{8 \pi V}{h^{3} c^{3}} \frac{k T}{l_{p}^{2}} \int_{0}^{\frac{1}{\sqrt{2}}} 2 x\left(1-x^{2}\right)^{\frac{1}{2}}\left(1-2 x^{2}\right) d x,
$$

and

$$
\bar{U} \propto \frac{8 \pi V}{h^{3} c^{3}} \frac{k T}{l_{p}^{3}} \int_{0}^{\frac{1}{\sqrt{2}}} 3 x^{2}\left(1-x^{2}\right)^{\frac{1}{2}}\left(1-2 x^{2}\right) d x,
$$

such that the energy of particles is bounded as we expect from the viewpoint of doubly special relativity

$$
\bar{\epsilon} \sim \frac{\bar{U}}{\bar{N}} \sim \frac{1}{l_{p}}
$$

which is independent of the temperature.
[1] G. Amelino-Camelia, Phys. Lett. B 510, 255 (2001) arXiv:hep-th/0012238.

[2] G. Amelino-Camelia, Int. J. Mod. Phys. D 11, 35 (2002) arXiv:gr-qc/0012051.

[3] G. Amelino-Camelia, J. Kowalski-Glikman, G. Mandanici and A. Procaccini, Int. J. Mod. Phys. A 20, 6007 (2005) arXiv:gr-qc/0312124.

[4] G. Amelino-Camelia, "The three perspectives on the quantum-gravity problem and their implications for the fate of Lorentz symmetry," arXiv:gr-qc/0309054.

[5] J. Magueijo and L. Smolin, Phys. Rev. Lett. 88, 190403 (2002) arXiv:hep-th/0112090.

[6] J. Magueijo and L. Smolin, Phys. Rev. D 67, 044017 (2003) arXiv:gr-qc/0207085.

[7] L. Smolin, "Quantum gravity with a positive cosmological constant," arXiv:hep-th/0209079.

[8] L. Smolin, Nucl. Phys. B 742, 142 (2006) arXiv:hep-th/0501091.

[9] G. Amelino-Camelia, M. Arzano, Y. Ling and G. Mandanici, Class. Quant. Grav. 23, 2585 (2006) arXiv:gr-qc/0506110.

[10] D. Colladay and V. A. Kostelecky, Phys. Rev. D 58, 116002 (1998) arXiv:hep-ph/9809521.

[11] S. R. Coleman and S. L. Glashow, Phys. Rev. D 59, 116008 (1999) arXiv:hep-ph/9812418.

[12] G. Amelino-Camelia and T. Piran, Phys. Rev. D 64, 036005 (2001) arXiv:astro-ph/0008107.

[13] T. Jacobson, S. Liberati and D. Mattingly, Phys. Rev. D 66, 081302 (2002) arXiv:hep-ph/0112207.

[14] R. C. Myers and M. Pospelov, Phys. Rev. Lett. 90, 211601 (2003) arXiv:hep-ph/0301124.

[15] T. A. Jacobson, S. Liberati, D. Mattingly and F. W. Stecker, Phys. Rev. Lett. 93, 021101 (2004) arXiv:astro-ph/0309681.

[16] J. Magueijo and L. Smolin, Class. Quant. Grav. 21, 1725
(2004) arXiv:gr-qc/0305055.

[17] P. Galan and G. A. Mena Marugan, Phys. Rev. D 70, 124003 (2004) arXiv:gr-qc/0411089.

[18] P. Galan and G. A. Mena Marugan, Phys. Rev. D 72, 044019 (2005) arXiv:gr-qc/0507098.

[19] J. Hackett, Class. Quant. Grav. 23, 3833 (2006) arXiv:gr-qc/0509103.

[20] R. Aloisio, A. Galante, A. Grillo, S. Liberati, E. Luzio and F. Mendez, Phys. Rev. D 73, 045020 (2006) arXiv:gr-qc/0511031.

[21] Y. Ling, X. Li and H. b. Zhang, "Thermodynamics of modified black holes from gravity's rainbow," arXiv:gr-qc/0512084.

[22] P. Galan and G. A. M. Marugan, Phys. Rev. D 74, 044035 (2006) arXiv:gr-qc/0608061.

[23] Y.Ling, S.He and H.Zhang, "The kinematics of particles moving in rainbow spacetime". arXiv:gr-qc/0609130.

[24] Y.Ling,B.Hu and X.Li, Phys. Rev. D 73 (2006) 087702, arXiv:gr-qc/0512083.

[25] R. J. Adler, P. Chen and D. I. Santiago, Gen. Rel. Grav. 33, 2101 (2001), P. Chen and R. J. Adler, Nucl. Phys. Proc. Suppl. 124, 103 (2003).

[26] S. Alexander and J. Magueijo, "Non-commutative geometry as a realization of varying speed of light." arXiv:hep-th/0104093.

[27] A. Ashtekar, T. Pawlowski and P. Singh, Phys. Rev. Lett. 96, 141301 (2006) arXiv:gr-qc/0602086.

[28] A. Ashtekar, T. Pawlowski and P. Singh, Phys. Rev. D 73, 124038 (2006) arXiv:gr-qc/0604013.

[29] P. Singh, K. Vandersloot and G. V. Vereshchagin, Phys. Rev. D 74, 043510 (2006) arXiv:gr-qc/0606032.

[30] It is worthwhile to point out that the naive identification involves more subtleties to clarify from the side of doubly special relativity. First the corresponding dispersion relation which has a higher order correction term 
does not manifestly provide an upper bound on either the momentum or energy of a single particle. Second the resulting density of states from (31) diverges as the energy approaches to the Planck scale, implying some cer- tain cutoff of the energy should be put in by hand which sounds not satisfying. 